\title{
Red blood cell distribution width and left ventricular mass index predict poor outcomes in patients with hypertrophic cardiomyopathy
}

\author{
Jia $\mathrm{Li}^{1}$, Weidong $\mathrm{Gao}^{1}$, Jinxue $\mathrm{Liu}^{1}$, Haifang Zhang${ }^{1}$, Jun Tao ${ }^{2}$, and Gaoxing Zhang ${ }^{1}$ \\ ${ }^{1}$ Jiangmen Central Hospital \\ ${ }^{2}$ Sun Yat-sen University First Affiliated Hospital
}

November 12, 2021

\begin{abstract}
Aim: To evaluate the prognostic utility of red blood cell distribution width (RDW) and left ventricular mass index (LVMI) in patients with hypertrophic cardiomyopathy (HCM). Patients \& methods: This study is a retrospective cohort analysis. Patients diagnosed with hypertrophic cardiomyopathy at the First Affiliated Hospital of Sun Yat-sen University from March 2014 to March 2019 were included. HCM patients were stratified into two groups based on the occurrence of major adverse cardiac events (MACE).Receiver operator characteristic (ROC) curves were then constructed and Cox regression models were employed to gauge the prognostic relevance of RDW and LVMI for HCM patients. Kaplan-Meier analysis evaluated the survival and MACE-free rate in patients with different level of RDW and LVMI. Results: A total of 300 patients with HCM were enrolled in this study and followed up for 40.56 \pm 18.33 months. Among them, 117 MACE (39.00\%), 40 all-cause deaths (13.33\%), 29 cardiovascular deaths (9.67\%). The level of RDW, LVMI, creatinine (Cr) and B-type pro-brain natriuretic peptide (NT-ProBNP) were statistically different between the MACE group and Non-MACE group $(P<0.05)$. Multivariate analysis showed that after adjusting for confounding factors, RDW and LVMI were independent predictors of all-cause mortality and MACE in HCM patients. ROC showed that $\mathrm{RDW}>0.13$ and $\mathrm{LVMI}>181 \mathrm{~g} / \mathrm{m}^{2}$ are the cut-off value to predict all-cause mortality and MACE. The AUC of the combination predicting the occurrence of all-cause mortality and MACE are 0.890 and 0.885 respectively. Kaplan-Meier analysis showed that the survival rate and MACE-free survival rate of group 1 (RDW[?]0.13 and LVMI[?]181g/m $\left.{ }^{2}\right)$ were significantly higher than group $2\left(\mathrm{RDW}>0.13\right.$ or $\left.\mathrm{LVMI}>181 \mathrm{~g} / \mathrm{m}^{2}\right)$, and group $3\left(\mathrm{RDW}>0.13\right.$ and $\left.\mathrm{LVMI}>181 \mathrm{~g} / \mathrm{m}^{2}\right)($ $P=0.000)$. Conclusion: We determined that increased RDW and LVMI was independently associated with MACE incidence and risk of mortality in HCM patients. Combined evaluation of RDW and LVMI yielded a more accurate predictive model of HCM patient outcomes relative to the use of either of these metrics in isolation. Our research can provide a theoretical basis in the occurrence of MACE for the high-risk HCM and intervene them properly and timely.
\end{abstract}

Red blood cell distribution width and left ventricular mass index predict poor outcomes in patients with hypertrophic cardiomyopathy

Running title: The predictive parameters in hypertrophic cardiomyopathy

Jia Li, MD ${ }^{\mathrm{a}, \mathrm{b}, \mathrm{c}++}$, Weidong Gao, MD ${ }^{\mathrm{a}++}$, Jinxue Liu, MD ${ }^{\mathrm{a}}$, Haifang Zhang, MD ${ }^{\mathrm{a}}$, Jun Tao, MD ${ }^{\mathrm{b},{ }^{*}}$, Gaoxing Zhang $\mathrm{MD}^{\mathrm{a}, *}$

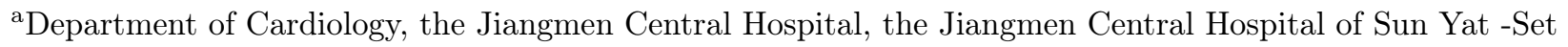
University, Jiangmen 529000, China

${ }^{\mathrm{b}}$ Department of Cardiology, the First Affiliated Hospital of Sun Yat-sen University, Guangzhou 510080, China 
'Sun Yat-sen University Zhongshan School of Medicine, Guangzhou 510000, China

${ }^{++} \mathrm{Jia} \mathrm{Li}$ and Weidong Gao contributed equally.

${ }^{*}$ Corresponding author.

Jun Tao, MD

Department of Cardiology, the First Affiliated Hospital of Sun Yat-sen University, No. 58, Zhongshan 2nd road, Yuexiu District, Guangzhou 510000, China.

E-mail address: taojungz123@163.com

Gaoxing Zhang, MD, Director,

Department of Cardiology, the Jiangmen Central Hospital, the Jiangmen Central Hospital of Sun Yat -Set University, No. 23, Beijie Road, Jiangmen, Guangdong 529000, China.

E-mail address: zhanggaoxing11@sohu.com

\section{Funding Sources}

This research did not receive any specific grant from funding agencies in the public, commercial, or not-forprofit sectors.

\section{Conflicts of Interest}

There are no conflicts of interest to declare.

\section{Acknowledgements}

Not applicable.

Red blood cell distribution width and left ventricular mass index predict poor outcomes in patients with hypertrophic cardiomyopathy

\section{Abstract}

Aim: To evaluate the prognostic utility of red blood cell distribution width (RDW) and left ventricular mass index (LVMI) in patients with hypertrophic cardiomyopathy (HCM).

Patients \& methods: This study is a retrospective cohort analysis. Patients diagnosed with hypertrophic cardiomyopathy at the First Affiliated Hospital of Sun Yat-sen University from March 2014 to March 2019 were included. HCM patients were stratified into two groups based on the occurrence of major adverse cardiac events (MACE).Receiver operator characteristic (ROC) curves were then constructed and Cox regression models were employed to gauge the prognostic relevance of RDW and LVMI for HCM patients. KaplanMeier analysis evaluated the survival and MACE-free rate in patients with different level of RDW and LVMI.

Results: A total of 300 patients with HCM were enrolled in this study and followed up for $40.56 \pm 18.33$ months. Among them, 117 MACE (39.00\%), 40 all-cause deaths (13.33\%), 29 cardiovascular deaths (9.67\%). The level of RDW, LVMI, creatinine $(\mathrm{Cr})$ and B-type pro-brain natriuretic peptide (NT-ProBNP) were statistically different between the MACE group and Non-MACE group $(P<0.05)$. Multivariate analysis showed that after adjusting for confounding factors, RDW and LVMI were independent predictors of allcause mortality and MACE in HCM patients. ROC showed that RDW $>0.13$ and LVMI $>181 \mathrm{~g} / \mathrm{m}^{2}$ are the cut-off value to predict all-cause mortality and MACE. The AUC of the combination predicting the occurrence of all-cause mortality and MACE are 0.890 and 0.885 respectively. Kaplan-Meier analysis showed that the survival rate and MACE-free survival rate of group 1 (RDW[?]0.13 and LVMI[?]181g/ $\mathrm{m}^{2}$ ) were significantly higher than group $2\left(\mathrm{RDW}>0.13\right.$ or LVMI $\left.>181 \mathrm{~g} / \mathrm{m}^{2}\right)$, and group $3\left(\mathrm{RDW}>0.13\right.$ and $\left.\mathrm{LVMI}>181 \mathrm{~g} / \mathrm{m}^{2}\right)(P$ $=0.000$ ). 
Conclusion: We determined that increased RDW and LVMI was independently associated with MACE incidence and risk of mortality in HCM patients. Combined evaluation of RDW and LVMI yielded a more accurate predictive model of HCM patient outcomes relative to the use of either of these metrics in isolation. Our research can provide a theoretical basis in the occurrence of MACE for the high-risk HCM and intervene them properly and timely.

Keywords: hypertrophic cardiomyopathy, red blood cell distribution, left ventricular mass index.

\section{Introduction}

Hypertrophic cardiomyopathy (HCM) is among the most prevalent forms of heritable cardiomyopathy, affecting between $1 / 200$ and $1 / 500$ of the overall general population. Treatment of HCM is generally ineffective, and the condition tends to steadily progress, ultimately resulting in the premature death of affected individuals [1-3]. While HCM affects both males and females equally and has been observed in diverse global populations, its clinical manifestations can vary substantially, ranging from asymptomatic cases to instances of sudden cardiac death (SCD) [2]. While SCD is a relatively rare outcome affecting $<1 \%$ of HCM patients per year, it is a particularly salient and concerning risk in those affected by this condition [3], particularly owing to the frequent coverage by the media of episodes of sudden cardiac arrest in competitive athletes [2] and to the difficulty of predicting SCD risk in those with HCM [4]. Several recent studies have sought to develop reliable approaches to identifying adults at an elevated risk of SCM. Known risk factors for HCM detected through these prior efforts have included family history of premature sudden death [5], unexplained syncope [6], non-sustained ventricular tachycardia (NSVT) [7], atypical changes in blood pressure during exercise (either a failure to rise by at least $20 \mathrm{mmHg}$ or a reduction by at least $20 \mathrm{mmHg}$ during exertion) [8], and severe left ventricular hypertrophy ([?]30 mm) [9]. While all of these predictors have been shown to exhibit a high negative predictive value ([?]90\%), they typically exhibit poor positive predictive value $(15-20 \%)$ [10]. It is thus urgent that novel approaches to reliably identifying predictors of HCM be identified in order to guide patient education and treatment as appropriate.

HCM is associated with a significant increase in cardiac mass, contributing to SCD, arrhythmias, and adverse left ventricular (LV) remodeling [11]. Left ventricular mass (LVM) in particular is linked to tissue fibrosis, and can be used to assess the overall degree of LV hypertrophy better than many other parameters, as hypertrophy patterns are often highly variable in regions distant from the maximal LV thickness [12]. Developments in echocardiographic technologies have clearly emphasized the utility of LVM in the management of HCM and associated cardiac hypertrophy, with LVM being negatively correlated with cardiovascular outcomes in both the general population and in those with hypertension/aortic stenosis [13], serving as an independent predictor of cardiovascular risk [14].

Red blood cell distribution width (RDW) is a parameter that can be measured through routine complete blood counts, assessing the overall variability of red blood cell (RBC) size distributions in a given individual [15]. Historically, RDW has primarily been monitored in the context of anemia differential diagnosis [16]. However, some studies have recently reported a strong independent relationship between RDW and adverse outcomes in patients with pulmonary arterial hypertension [17], coronary disease [16], and heart failure [18]. The prognostic relevance of RDW in HCM patients, however, remains to be studied in depth. In one analysis, baseline RDW was shown to be positively associated with heart failure incidence in HCM patients without a history of such failure [19]. The relationship between RDW and other negative outcomes including cardiac

death and mortality is unclear at present. As such, this study was conducted to evaluate the relevance of RDW and LVMI alone and in combination as predictors of negative cardiac outcomes and all-cause death in HCM patients.

\section{Patients \& Methods}

\subsection{Patients}

This study is a retrospective cohort analysis. Patients who were diagnosed with hypertrophic cardiomyopathy at the First Affiliated Hospital of Sun Yat-sen University from March 2014 to March 2019 were included 
. The criteria of inclusion into the study were 1) age > 18 years; 2) diagnosis of HCM according to the European Society of Cardiology (ESC) Guidelines [10]. The exclusion criteria for the study were : 1)incomplete information; 2)Lost to follow-up; 3) Left ventricular hypertrophy contributed by other disease like hypertension, aortic stenosis and congenital subaortic diaphragm, etc. All participants provided written informed. This study was consistent with the Declaration of Helsinki, and was approved by our hospital ethics committee.

\subsection{Data collection and outcome}

Venous plasma hemoglobin (Hb), hematocrit (HCT), mean corpuscular volume (MCV), mean platelet volume (MPV), platelet distribution width (PDW), platelet (PLT), C reactive protein (CRP), creatinine $(\mathrm{Cr})$, serum albumin (ALB), N-terminal pro-natriuretic peptide (NT-proBNP), troponin, uric acid (UA), thyroidstimulating hormone (TSH), and RDW levels were evaluated using standard biochemical approaches in our clinical laboratory department. Two researchers blinded to patient RDW and clinical outcomes independently analyzed all echocardiographic examinations and measured left atrial diameter (LAD), maximum left ventricular wall thickness (MLVWT), left ventricular end-diastolic diameter (LVEDD), and interventricular septum diameter (IVSD). LVM was measured using the Devereux equation [20]. LVM $=0.8(1.04$ ((IVST + LVEDD + PWT)3 LVEDD3) $+0.6 \mathrm{~g}$, with LVMI being determined via dividing LVM by body surface area. LVEF was assessed via the biplane Simpson method [21]. Dynamic left ventricular outflow tract obstruction (LVOTO) was assessed via continuous-wave Doppler and estimated with the simplified Bernoulli equation [22].

\subsection{Outcomes and follow-up}

The primary end-point was all-cause mortality, including cardiac death and non-cardiac death [3]. The secondary endpoint was major adverse cardiac events ( MACE). MACE defined as all-cause mortality, the development of symptomatic congestive heart failure, admission for heart failure, fatal arrhythmia (ventricular tachycardia (VT) and ventricular fibrillation (VF)) and the composite of cardiac death from heart failure, malignant arrhythmia, and SCD [2]. A medical recorder with no information regarding a given patient's laboratory findings or treatment reviewed all MACE. The endpoint for follow-up was the date of first MACE incidence and was determined through hospital medical records. Telephone interviews and other approaches were used to follow discharged patients through November 30, 2020.

\subsection{Statistical analysis}

RDW cutoff values were used to stratify patients into two groups. Data normality was assessed via the Kolmogorov-Smirnov test, with normally and non-normally distributed continuous values being given as means with standard deviations and medians with interquartile ranges (IQRs), respectively, whereas categorical data were given as numbers (frequencies). These three data types were compared via Student's t-tests, Mann-Whitney U tests, and chi-squared tests, respectively. Spearman correlation analyses were conducted to assess the association between RDW and LVMI.

The Kaplan-Meier approach was employed to construct survival curves, with data being censored at the more recent follow-up time point if patients failed to reach the pertinent endpoints. Predictors of mortality in HCM patients were identified through univariate and multivariate Cox regression analyses, with the multivariate model being designed to control for potential confounding variables via incorporating them into this analysis.

Results were given as hazard ratios (HRs) and 95\% confidence intervals (CIs). The prognostic relevance of RDW was then further evaluated by comparing outcomes in patients stratified according to the chosen RDW cutoff value using Kaplan-Meier curves.

Area under the receiver operating characteristic (ROC) curve (AUC) values were used to compare the relative prognostic relevance of RDW alone to that of RDW + LVMI. DeLong's test was utilized to compare AUC values for these models [23], with calculations being conducted using MedCalc v.19.0.7. $\mathrm{P}<0.05$ was the significance threshold for this study, and SPSS v25.0 (IBM Corp, NY, USA) was used for statistical testing herein. 


\section{Results}

\subsection{Comparison of general baseline data between MACE group and non-MACE group}

Over a median $40.56+-18.33$ month follow-up period, 30/357 patients $(8.40 \%)$ were lost to follow-up. Those with incomplete data $(\mathrm{n}=3)$ and patients $<18$ years $(\mathrm{n}=24)$ were excluded from this report. Finally, 300 HCM patients were included in our final analyses. Of these patients, 39.00\% (117/300) suffered from a MACE, including 40 cases of all-cause death, 29 cases of cardiovascular mortality. Patients were thus separated into a MACE group and a non-MACE group (183 and 117 patients, respectively). Compared to those in the non-MACE group, patients in the MACE group were older, and had higher rates of coronary artery disease $(\mathrm{CAD})$ and atrial fibrillation $(\mathrm{AF})$. Individuals in the MACE group also exhibited lower Hb, HCT, and MCHC but higher serum Cr, NT-proBNP, LVMI and RDW levels compared to those in the non-MACE group $(P<0.05)$. (Table 1)

\subsection{RDW and LVMI can independently predicts all -cause mortality and MACE}

Cox regression analyses revealed that RDW (HR 1.877; 95\% CI 1.277-2.533; $P=0.004$ ), LVMI (HR 2.347; $95 \%$ CI $1.318-4.669 ; P=0.000)$ were all predictive of all-cause mortality after adjusting for potential confounding factors. Besides, RDW (HR 1.013; 95\% CI 1.009-1.017; $P=0.000$ ), LVMI (HR 1.715; 95\% CI $1.211-2.519 ; P=0.000)$ can independently predict the occurrence of MACE in patients with HCM.(Table 2)

\subsection{ROC curve analysis}

We next constructed ROC curves using RDW, LVMI, and a combination of LVMI and RDW to predict HCM patient outcomes (Fig. 1). The cut-off value of RDW for the all -cause mortality and MACE was 0.13, and the AUC was 0.753 and 0.746 respectively. For LVMI, the AUC for predicting all-cause mortality and MACE incidence was 0.855 and 0.838 respectively. When RDW and LVMI were evaluated together, the AUC value was $0.890(95 \%$ CI $0.849-0.923, P<0.01)$ in predicting the occurrence of all-cause mortality, and was 0.885 (95\% CI 0.844-0.919, $P<0.01)$ in predicting the occurrence of MACE. As such, the combined evaluation of RDW and LVMI was superior to either of these variables alone when predicting the risk of all-cause mortality and MACE in patients with HCM. (Table 3).

\subsection{Relationship between survival and the level of RDW and LVMI in HCM patients}

According to whether RDW $>0.13$ and $\mathrm{LVMI}>181 \mathrm{~g} / \mathrm{m}^{2}$, the patients were divided into 3 groups, including group 1 (RDW[?]0.13 and LVMI[?]181g/m2), group $2(\mathrm{RDW}>0.13$ or $\mathrm{LVMI}>181 \mathrm{~g} / \mathrm{m} 2)$ and group 3 $($ RDW $>0.13$ and LVMI $>181 \mathrm{~g} / \mathrm{m} 2)$. The results showed that there were statistically different in the occurrence of all-cause mortality and MACE among the groups.(Table 4).Kaplan-Meier analysis showed that the survival rate of group 1 was significantly higher than that of group 2 and group 3 (98.36\% vs. 92.86 vs. $54.55 \%, \mathrm{P}=0.000$ ), as shown in Fig. 2a . Besides, the MACE-free survival rate of group 1 was significantly higher than that of group 2 and group 3 (86.89 vs. 62.50 vs. $15.15 \%, P=0.000$ ) as shown in Fig. 2b .

\section{Discussion}

Herein, we determined that a higher baseline RDW was independently associated with the risk of cardiac death, heart failure, and all-cause mortality in a population of HCM patients with RDW values that largely fell within the normal range. In addition, we detected a significant correlation between LVMI and RDW, and we determined that the simultaneous evaluation of both RDW and LVMI was better able to predict adverse events in HCM patients than the evaluation of RDW alone. LV hypertrophy is thus closely associated with high rates of heart failure and mortality among individuals with HCM.

A range of different laboratory parameters have been highlighted as putative markers of inflammation and cardiovascular disease in recent years. For example, uric acid [24], NT-proBNP [25], and troponin [26] have all been previously associated with adverse HCM patient outcomes. Herein, we found higher RDW values to be linked to increases in NT-proBNP and CRP but to decreases in hemoglobin and serum albumin levels, consistent with a higher RDW being indicative of sicker patients. Even after adjusting for a range of 
potential confounding variables, we found that higher RDW values remained linked to negative outcomes in these HCM patients, underscoring the prognostic utility of this hemodynamic parameter. Importantly, RDW values can be easily established based on the results of a patient's complete blood count, and thus require no additional medical testing. We also found that the combined evaluation of RDW and LVMI was able to better predict long-term cardiovascular outcomes in HCM patients, indicating that these two predictors may be ideal targets for efforts to better prevent, manage, and treat this dangerous condition.

We found that higher RDW values were linked to clinically significant increases in HCM patient risk of mortality, thus expanding on the results of two prior studies. In a preclinical study, Stanzani et al. had demonstrated that an increase in RDW was independently predictive of increased cardiac mortality in HCM model cats [27]. A separate study of $98 \mathrm{HCM}$ patients with no history of heart failure found RDW to be independently linked to heart failure onset. In this study, we analyzed HCM patients with and without anemia and/or heart failure, and we did not specifically restrict our outcome measurements to heart failure incidence. By expanding the scope of our analyses, we were thereby able to establish RDW as a powerful independent predictor of diverse negative clinical outcomes in HCM patients.

While the mechanistic link between increased RDW values and negative HCM patient outcomes remains to be clarified, there are linked several factors contributing to this relationship. For one, increases in RDW may correspond to folate or vitamin B12 deficiencies [28]. RDW values for most patients in the present study, however, were within the normal range, and we adjusted results for mean corpuscular volume which is correlated with folate and vitamin B12 levels. As such, while we did not directly evaluate patient nutritional status, our results are not consistent with such a model. Second, thrombosis may contribute to the relationship between HCM and RDW given that declining RBC functionality has been previously linked to an increased risk of RBC adherence to endothelial cells and consequent thrombus formation [29]. RDW has reportedly been linked to prothrombotic activity through renin-angiotensin system (RAS) activation through angiotensin II type 1a receptor [22]. Such increases in RAS activating can in turn drive cellular proliferation and hypertrophy, impacting LV hypertrophy and remodeling and thereby contributing to negative outcomes [19]. Third, inflammatory cytokine production may further explain the relationship between RDW and HCM patient outcomes, given the well-documented importance of these cytokines in the context of HCM pathogenesis. Such proinflammatory factors can suppress erythropoietin-induced erythrocyte maturation, which can be partially reflected by a rise in RDW. As such, higher RDW values may reflect increases in underlying inflammation that are associated with poor clinical outcomes [30]. This may thus represent a key mechanism linking high RDW and high rates of negative clinical outcomes in individuals with HCM. There is also a purported link between RDW and abnormal renal functionality [31], although we detected no association between this hemodynamic parameter and serum creatinine levels. Overall, we hypothesize that increases in RDW may correspond to more severe inflammation and risk of thrombosis, thus contributing to a poor HCM patient prognosis. However, future work will be needed to formally test this hypothesis and to more firmly establish the determinants of RDW in those with HCM.

Left ventricular hypertrophy (LVH) is a potential consequence of cardiovascular disease that has been independently linked to SCD risk in individuals diagnosed with HCM. Prior work suggests that RDW is closely linked to specific echocardiographic parameters used to assess LVH in patients with essential hypertension [32], chronic kidney disease [33], and atrial fibrillation [34]. Similarly, we identified a positive relationship between LVMI and RDW in individuals with HCM, indicating that RDW may be a biomarker of LVH. Oxidative stress may explain the relationship between these two variables, as Cave et al. [35] previously showed that angiotensin II, endothelin-1, tumor necrosis factor (TNF), and pulsatile mechanical stretch were able to promote symptoms of cardiac hypertrophy in vitro that were linked to intracellular reactive oxygen species production. Antioxidants can inhibit these pathogenic processes, and depleting endogenous antioxidants can result in an increased RDW [35].

Our article has several strengths. For one, this study was the first to evaluate overall adverse clinical outcomes in HCM patients, with these outcomes having been assessed based on predefined criteria without knowledge regarding patient RDW levels. However, there are also certain limitations to this approach. For one, we are 
unable to exclude the potential for residual confounding variables having impacted our analysis given that this study was observational in nature. Even so, we hypothesized the existence of a relationship between RDW levels and adverse outcomes prior to beginning our analyses, thus reducing the risk of erroneous conclusions. We additionally adjusted our analyses for potential confounding variables including clinical and laboratory parameters that were linked to RDW levels in our patient cohort. This study is also limited by the fact that it was a single-center analysis of Chinese patients with HCM previously diagnosed at the First Affiliated Hospital of Sun Yat-sen University during a specific period of time, and so these results may not be generalizable to the general population or to other ethnic groups. We also observed a lower rate of family history of HCM in our patient cohort relative to previously reported rates in epidemiological studies of HCM, which may be attributable to the majority of these HCM patients exhibiting an asymptomatic presentation and not having undergone routine physical examinations in the hospital, thus preventing them from providing a detailed family history. Our study also did not provide any evidence regarding specific clinical approaches that can be taken following the detection of HCM patients with high RDW levels. Additional multicenter randomized controlled trials and genetic studies will thus be essential in order to more fully understand the mechanistic basis for HCM and to identify appropriate approaches that can be taken to treat or manage HCM patients with high RDW.

\section{Conclusion}

In conclusion, we found that both RDW and LVMI are independent predictors of HCM patient outcomes. A combination of these two metrics was of greater prognostic value as a means of predicting adverse outcomes in these patients.

\section{References}

[1] Tuohy CV, Kaul S, Song HK, Nazer B, Heitner SB. Hypertrophic cardiomyopathy: the future of treatment. Eur J Heart Fail 2020;22(2):228-40.

[2] Ozawa K, Funabashi N, Takaoka H, Kobayashi Y. Successful MACE risk stratification in hypertrophic cardiomyopathy patients using different 2D speckle-tracking TTE approaches. Int J Cardiol. 2017. 228: $1015-1021$.

[3] Lorenzini M, Anastasiou Z, O'Mahony C, et al. Mortality Among Referral Patients With Hypertrophic Cardiomyopathy vs the General European Population. JAMA Cardiol. 2020. 5(1): 73-80.

[5] Flenner F, Jungen C, Kupker N, et al. Translational investigation of electrophysiology in hypertrophic cardiomyopathy. J Mol Cell Cardiol. 2021. 157: 77-89.

[6] Huurman R, Schinkel A, van der Velde N, et al. Effect of body surface area and gender on wall thickness thresholds in hypertrophic cardiomyopathy. Neth Heart J. 2020. 28(1): 37-43.

[7] McLELlan AJ, Ellims AH, Prabhu S, et al. Diffuse Ventricular Fibrosis on Cardiac Magnetic Resonance Imaging Associates With Ventricular Tachycardia in Patients With Hypertrophic Cardiomyopathy. J Cardiovasc Electrophysiol. 2016. 27(5): 571-80.

[8] Smith ED, Tome J, Mcgrath R, et al. Exercise hemodynamics in hypertrophic cardiomyopathy identify risk of incident heart failure but not ventricular arrhythmias or sudden cardiac death. Int J Cardiol. 2019. 274: 226-231.

[9] Spirito P, Bellone P, Harris KM, Bernabo P, Bruzzi P, Maron BJ. Magnitude of left ventricular hypertrophy and risk of sudden death in hypertrophic cardiomyopathy. N Engl J Med 2000;342(24):1778-85.

[10] Elliott PM, Anastasakis A, Borger MA, et al. 2014 ESC Guidelines on diagnosis and management of hypertrophic cardiomyopathy: the Task Force for the Diagnosis and Management of Hypertrophic Cardiomyopathy of the European Society of Cardiology (ESC). Eur Heart J. 2014. 35(39): 2733-79.

[11] Musumeci B, Tini G, Russo D, et al. Left Ventricular Remodeling in Hypertrophic Cardiomyopathy: An Overview of Current Knowledge. J Clin Med. 2021. 10(8). 
[12] Losi MA, Imbriaco M, Canciello G, Pacelli F, Di Nardo C, Lombardi R, et al. Left ventricular mass in hypertrophic cardiomyopathy assessed by $2 \mathrm{~d}$-echocardiography: validation with magnetic resonance imaging. J Cardiovasc Transl Res 2020;13(2):238-44.

[13] Losi MA, Imbriaco M, Canciello G, et al. Left Ventricular Mass in Hypertrophic Cardiomyopathy Assessed by 2D-Echocardiography: Validation with Magnetic Resonance Imaging. J Cardiovasc Transl Res. 2020. 13(2): 238-244.

[14] Sreedharan S, Mathew A, Paul Z, et al. Changes in Left Ventricular Mass and Cardiovascular Risk Factors after Kidney Transplantation. Saudi J Kidney Dis Transpl. 2020. 31(5): 1006-1013.

[15]Beydoun MA, Shaked D, Hossain S, et al. Red cell distribution width, anemia and their associations with white matter integrity among middle-aged urban adults. Neurobiol Aging. 2021. 105: 229-240.

[16] Tonelli M, Sacks F, Arnold M, Moye L, Davis B, Pfeffer M, et al. Relation between red blood cell distribution width and cardiovascular event rate in people with coronary disease. Circulation 2008;117(2):163-8.

[17] Ulrich A, Wharton J, Thayer TE, Swietlik EM, Assad TR, Desai AA, et al. Mendelian randomisation analysis of red cell distribution width in pulmonary arterial hypertension. Eur Respir J 2020;55(2):1901486.

[18] Xanthopoulos A, Papamichalis M, Zajichek A, Milinovich A, Kattan MW, Skoularigis J, et al. In-hospital red blood cell distribution width change in patients with heart failure. Eur J Heart Fail 2019;21(12):1659-61.

[19] Yang HJ, Liu X, Qu C, Shi SB, Liang JJ, Yang B. Usefulness of red blood cell distribution width to predict heart failure hospitalization in patients with hypertrophic cardiomyopathy. Int Heart J 2018;59(4):779-85.

[20] Devereux RB, Alonso DR, Lutas EM, Gottlieb GJ, Campo E, Sachs I, et al. Echocardiographic assessment of left ventricular hypertrophy: comparison to necropsy findings. Am J Cardiol 1986;57(6):450-8.

[21] Lang RM, Bierig M, Devereux RB, Flachskampf FA, Foster E, Pellikka PA, et al. Recommendations for chamber quantification. Eur J Echocardiogr 2006;7(2):79-108.

[22] Nakatani S, Marwick TH, Lever HM, Thomas JD. Resting echocardiographic features of latent left ventricular outflow obstruction in hypertrophic cardiomyopathy. Am J Cardiol 1996;78(6):662-7.

[23] DeLong ER, DeLong DM, Clarke-Pearson DL. Comparing the areas under two or more correlated receiver operating characteristic curves: a nonparametric approach. Biometrics 1988;44(3):837-45.

[24] Zhu L, Wang J, Wang Y, Jia L, Sun K, Wang H, et al. Plasma uric acid as a prognostic marker in patients with hypertrophic cardiomyopathy. Can J Cardiol 2015;31(10):1252-8.

[25] Meng XB, Wang WY, Zhang K, Qi Y, An SM, Wang SY, et al. Value of N-terminal pro-B-type natriuretic peptide on long-term outcome of patients with hypertrophic cardiomyopathy. Zhonghua Xin Xue Guan Bing Za Zhi 2018;46(3):192-7. (in Chinese)

[26] Hasler S, Manka R, Greutmann M, Gamperli O, Schmied C, Tanner FC, et al. Elevated high-sensitivity troponin $\mathrm{T}$ levels are associated with adverse cardiac remodelling and myocardial fibrosis in hypertrophic cardiomyopathy. Swiss Med Wkly 2016;146:w14285.

[27] Stanzani G, Cowlam R, English K, Connolly DJ. Evaluation of red blood cell distribution width in cats with hypertrophic cardiomyopathy. J Vet Cardiol 2015;17:S233-43.

[28] Viswanath D, Hegde R, Murthy V, Nagashree S, Shah R. Red cell distribution width in the diagnosis of iron deficiency anemia. Indian J Pediatr 2001;68(12):1117-9.

[29] D'Alessandro A, Blasi B, D'Amici GM, Marrocco C, Zolla L. Red blood cell subpopulations in freshly drawn blood: application of proteomics and metabolomics to a decades-long biological issue. Blood Transfus 2013;11(1):75-87. 
[30] Tsimikas S, Willerson JT, Ridker PM. C-reactive protein and other emerging blood biomarkers to optimize risk stratification of vulnerable patients. J Am Coll Cardiol 2006;47:C19-31.

[31] Okonko DO, Marley SB, Anker SD, Poole-Wilson PA, Gordon MY. Suppression of erythropoiesis in patients with chronic heart failure and anaemia of unknown origin: evidence of an immune basis. Int $\mathrm{J}$ Cardiol 2013;166(3):664-71.

[32] Chen L, Li Z, Li Y, Xue J, Chen P, Yan S, et al. Red cell distribution width and inappropriateness of left ventricular mass in patients with untreated essential hypertension. PLoS One 2015;10(3):e0120300.

[33] Gromadziński L, Januszko-Giergielewicz B, Pruszczyk P. Red cell distribution width is an independent factor for left ventricular diastolic dysfunction in patients with chronic kidney disease. Clin Exp Nephrol 2015;19(4):616-25.

[34] Yao HM, Wang XL, Peng X, Chen SY, Wan X, Zuo W, et al. Increased red blood cell distribution width might predict left ventricular hypertrophy in patients with atrial fibrillation. Medicine (Baltimore). 2020;99(37):e22119.

[35] Tse G, Yan BP, Chan YW, Tian XY, Huang Y. Reactive oxygen species, endoplasmic reticulum stress and mitochondrial dysfunction: the link with cardiac arrhythmogenesis. Front Physiol 2016;7:313.

\section{Figure Legends}

Fig. 1. ROC showed the predictive value of RDW and LVMI alone in combination to predict the occurrence of all-cause mortality ( a) and MACE (b) in HCM patients.

Fig. 2 Kaplan-Meier curves comparing the rates of mortality-free survival (a), and MACE-free survival (b), among group $1\left(\mathrm{RDW}[?] 0.13\right.$ and LVMI[?]181g $\left./ \mathrm{m}^{2}\right)$, group $2\left(\mathrm{RDW}>0.13\right.$ or $\left.\mathrm{LVMI}>181 \mathrm{~g} / \mathrm{m}^{2}\right)$ and group $3\left(\right.$ RDW $>0.13$ and $\left.\mathrm{LVMI}>181 \mathrm{~g} / \mathrm{m}^{2}\right)$

Table 1. Characteristics of hypertrophic cardiomyopathy with or without a MACE.

\begin{tabular}{|c|c|c|c|c|c|}
\hline Variable & Variable & Without MACE $(\mathrm{n}=183)$ & With MACE $(\mathrm{n}=117)$ & $\mathrm{t} / \chi^{2}$ & $P \mathrm{v}$ \\
\hline \multirow[t]{9}{*}{ Demographics } & Demographics & & & & \\
\hline & Age (years) & $54.97 \pm 13.19$ & $65.14 \pm 12.61$ & -6.626 & 0.0 \\
\hline & Female sex $(\%)$ & $76(41.53)$ & $54(46.15)$ & 0.621 & 0.4 \\
\hline & $\mathrm{SBP}(\mathrm{mm} \mathrm{Hg})$ & $126.33 \pm 13.19$ & $125.42 \pm 13.27$ & 0.582 & .5 \\
\hline & DBP (mm Hg) & $77.67 \pm 3.19$ & $77.54 \pm 3.02$ & 0.351 & .7 \\
\hline & Family history of HCM (\%) & $10(5.46)$ & $7(5.98)$ & 0.036 & .8 \\
\hline & Coronary artery disease $(\%)$ & $8(6.8)$ & $15(18.8)$ & 7.197 & .0 \\
\hline & Diabetes mellitus (\%) & $31(16.94)$ & $19(16.24)$ & 0.025 & 0.8 \\
\hline & Atrial fibrillation (\%) & $20(11.17)$ & $24(20.42)$ & 5.238 & 0.0 \\
\hline \multirow[t]{14}{*}{ Laboratory parameters } & Laboratory parameters & & & & \\
\hline & $\mathrm{Hb}(\mathrm{g} / \mathrm{l})$ & $145.12 \pm 10.29$ & $142.03 \pm 11.47$ & 2.425 & \\
\hline & $\operatorname{HCT}(\%)$ & $0.43 \pm 0.02$ & $0.39 \pm 0.05$ & 9.685 & \\
\hline & RDW content (per \%) & $0.13 \pm 0.01$ & $0.14 \pm 0.01$ & 8.448 & 0.0 \\
\hline & $\mathrm{MCV}(\mathrm{fl})$ & $89.10 \pm 3.29$ & $88.40 \pm 3.03$ & 1.853 & 0.0 \\
\hline & $\mathrm{MCHC}(\mathrm{g} / \mathrm{l})$ & $340.28 \pm 9.86$ & $332.04 \pm 11.65$ & 6.572 & 0.0 \\
\hline & $\operatorname{MPV}(\mathrm{f})$ & $10.14 \pm 1.04$ & $9.91 \pm 1.59$ & 1.515 & 0.1 \\
\hline & PDW (umol/l) & $13.07 \pm 3.04$ & $12.87 \pm 2.03$ & 0.628 & 0.5 \\
\hline & PLT,$\times 109 / 1$ & $215.42 \pm 42.37$ & $219.94 \pm 47.15$ & 0.862 & 0.38 \\
\hline & CRP (mg/l) & $9.04 \pm 4.86$ & $10.02 \pm 5.76$ & 1.583 & 0.1 \\
\hline & Creatinine $(\mu \mathrm{mol} / \mathrm{l})$ & $94.33 \pm 12.86$ & $103.12 \pm 15.37$ & 5.346 & 0.0 \\
\hline & $\operatorname{ALB}(g / l)$ & $38.42 \pm 3.07$ & $38.31 \pm 3.57$ & 0.284 & $0.7^{\prime}$ \\
\hline & NT-proBNP $(\mathrm{pg} / \mathrm{ml})$ & $632.43 \pm 411.45$ & $2066.62 \pm 1135.45$ & 15.574 & \\
\hline & $\mathrm{UA}(\mathrm{mg} / \mathrm{l})$ & $320.60 \pm 104.17$ & $325.01 \pm 128.68$ & 0.326 & \\
\hline
\end{tabular}


Echocardiography

Echocardiography

LAD $(\mathrm{mm})$
LVEDD $(\mathrm{mm})$
IVSD $(\mathrm{mm})$
MLVWT $(\mathrm{mm})$
LVEF $(\%)$
LVMI $\left(\mathrm{g} / \mathrm{m}^{2}\right)$
LVOTO $(\%)$

$40.11 \pm 4.57$

$46.18 \pm 5.76$

$17.25 \pm 3.02$

$19.32 \pm 3.57$

$65.38 \pm 4.21$

$141.42 \pm 37.22$

101 (55.19)
$40.27 \pm 4.34$
$45.87 \pm 5.96$
$17.32 \pm 3.17$
$21.44 \pm 3.12$
$66.15 \pm 4.22$
$201.14 \pm 47.19$
$77(65.81)$

0.302

0.449

0.192

5.487

15.437

12.190

3.337
0.763

0.654

0.848

0.000

0.124

0.000

0.068

All values are expressed as mean \pm standard deviation or $n(\%)$ or medians with interquartile ranges.

${ }^{a}$ Statistically significant.

6

Table 2. COX regression analysis shows that RDW and LVMI can independently predict the occurrence of all-cause mortality and MACE in patients with HCM

\begin{tabular}{lllllll}
\hline & & Model 1 & Model 1 & Model 2 & Model 2 & Model 3 \\
All-cause mortality & All-cause mortality & HR $(95 \% \mathrm{CI})$ & $P$ value & HR $(95 \% \mathrm{CI})$ & $P$ value & HR (95\% C \\
& RDW content (per \%) & $3.016(1.512-5.337)$ & 0.000 & $2.821(1.317-4.572)$ & 0.000 & $1.887(1.277$ \\
& LVMI $\left(\mathrm{g} / \mathrm{m}^{2}\right)$ & $3.776(1.727-8.256)$ & 0.000 & $3.123(1.513-6.725)$ & 0.000 & $2.347(1.318$ \\
MACE & & & & & \\
& MACE & & & & & \\
& RDW content (per \%) & $2.136(1.444-3.159)$ & 0.000 & $1.704(1.133-2.778)$ & 0.000 & $1.013(1.009$ \\
& LVMI $\left(\mathrm{g} / \mathrm{m}^{2}\right)$ & $2.629(1.788-3.865)$ & 0.000 & $1.874(1.278-2.633)$ & 0.000 & $1.715(1.211$ \\
\hline
\end{tabular}

Model 1: Unadjusted confounding factors. Model 2: Adjusted for confounding factors such as age, gender, coronary heart disease and atrial fibrillation. Model 3: In addition to Model 2, factors such as Hb, HCT, MCHC, Cr, and NT-ProBNP are also adjusted.

Table 3. The value of RDW and LVMI alone and in combination in predicting all-cause mortality and MACE in HCM patients

\begin{tabular}{llllllll}
\hline Variable & Variable & Cut-off value & AUC & $95 \%$ CI & Sensitivity & Specificity & You-den index \\
All-cause mortality & All-cause mortality & & & & & & \\
& RDW & $>0.13$ & 0.753 & $0.701-0.801$ & 80.00 & 61.92 & 0.4192 \\
& LVMI & $>181 \mathrm{~g} / \mathrm{m}^{2}$ & 0.855 & $0.810-0.893$ & 87.50 & 73.08 & 0.6058 \\
MACE & combination & $>0.63894$ & 0.890 & $0.849-0.923$ & 95.00 & 81.92 & 0.7692 \\
& MACE & & & & & & \\
& RDW & $>0.13$ & 0.746 & $0.693-0.794$ & 66.67 & 71.04 & 0.3770 \\
& LVMI & $>181 \mathrm{~g} / \mathrm{m}^{2}$ & 0.838 & $0.792-0.878$ & 70.94 & 83.06 & 0.5400 \\
& combination & $>0.5189$ & 0.885 & $0.844-0.919$ & 73.50 & 90.16 & 0.6367 \\
\hline
\end{tabular}

Table 4. Comparison of risk of all-cause mortality and MACE in Group 1, Group 2 and Group 3

\begin{tabular}{llllllll}
\hline Variable & Variable & Group 1(n=122) & Group 2(n=112) & Group 3(n=66) & $P_{1-2}$ & $P_{1-3}$ & $P_{2-3}$ \\
All-cause mortality & All-cause mortality & $2(1.64)$ & $8(7.14)$ & $30(45.45)$ & 0.000 & 0.000 & 0.000 \\
& Non-mortality & $120(98.36)$ & $104(92.86)$ & $36(54.55)$ & - & - & - \\
MACE & MACE & $16(13.11)$ & $42(37.50)$ & $56(84.85)$ & 0.000 & 0.000 & 0.000 \\
& Non-MACE & $106(86.89)$ & $70(62.50)$ & $10(15.15)$ & & & \\
\hline
\end{tabular}


Group 1: the patients with RDW[?]0.13 and LVMI[?]181g/m2. Group 2: the patients with RDW $>0.13$ or LVMI $>181 \mathrm{~g} / \mathrm{m} 2$. Group $3:$ the patients with RDW $>0.13$ and LVMI $>181 \mathrm{~g} / \mathrm{m} 2$

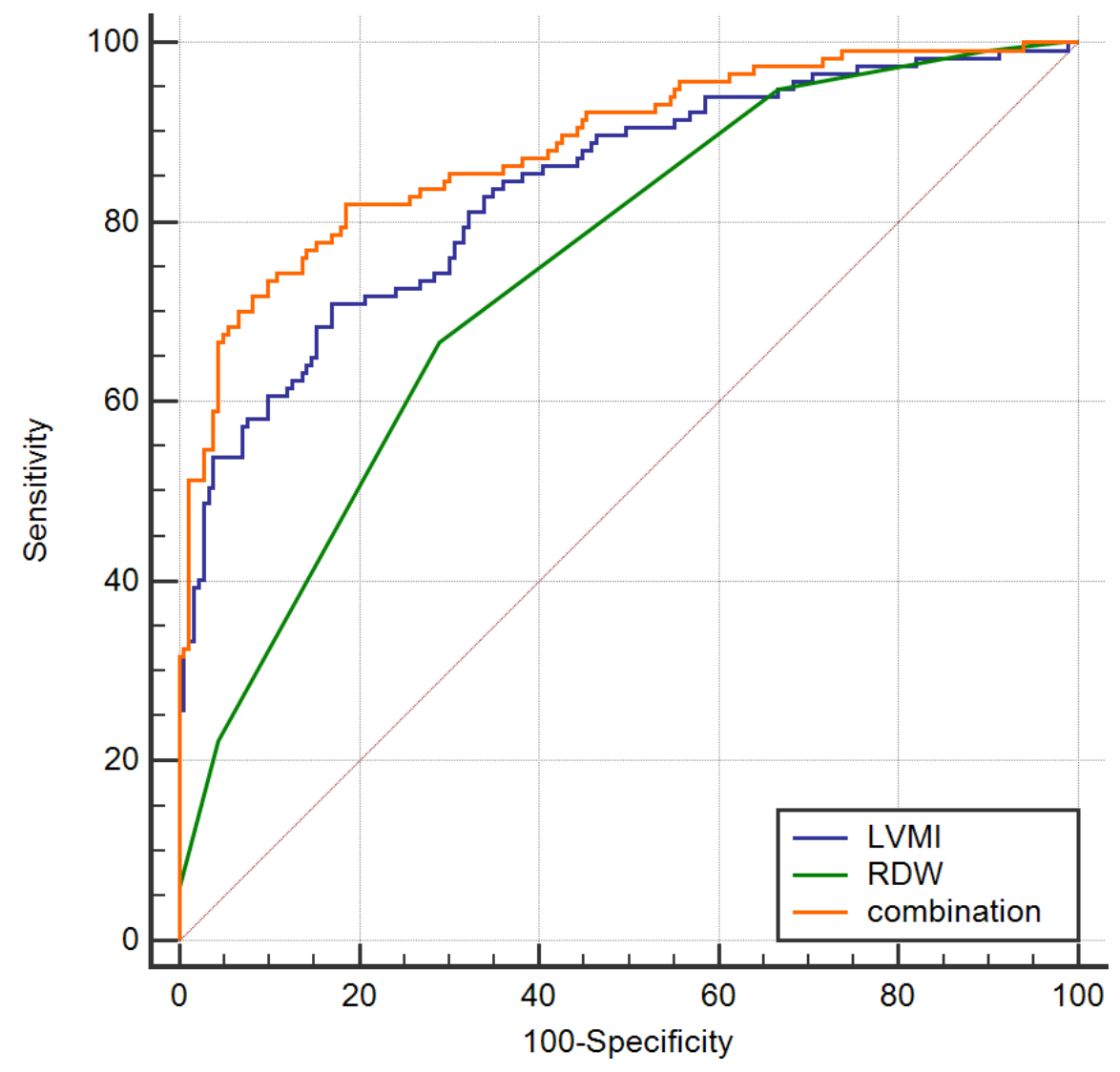



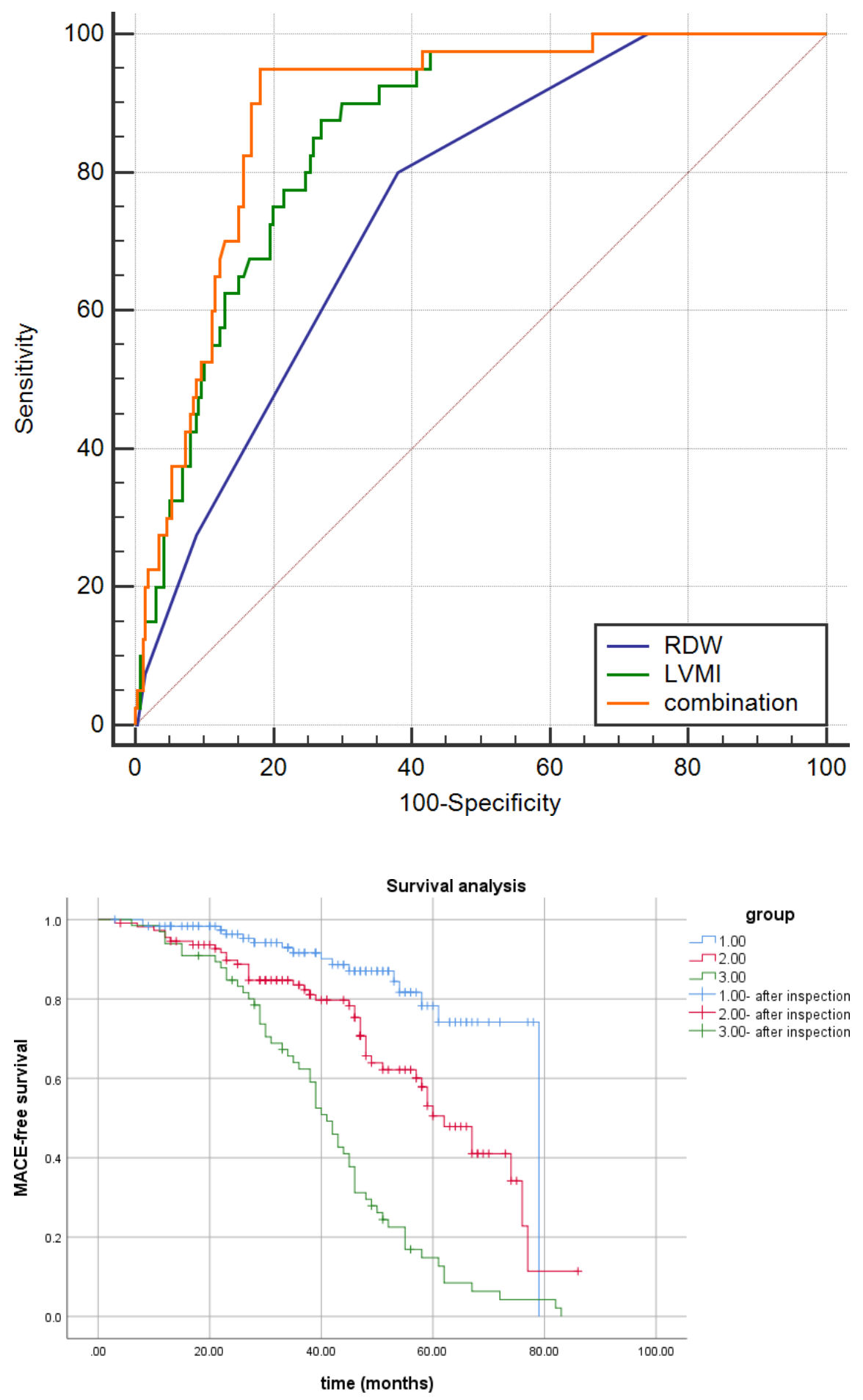


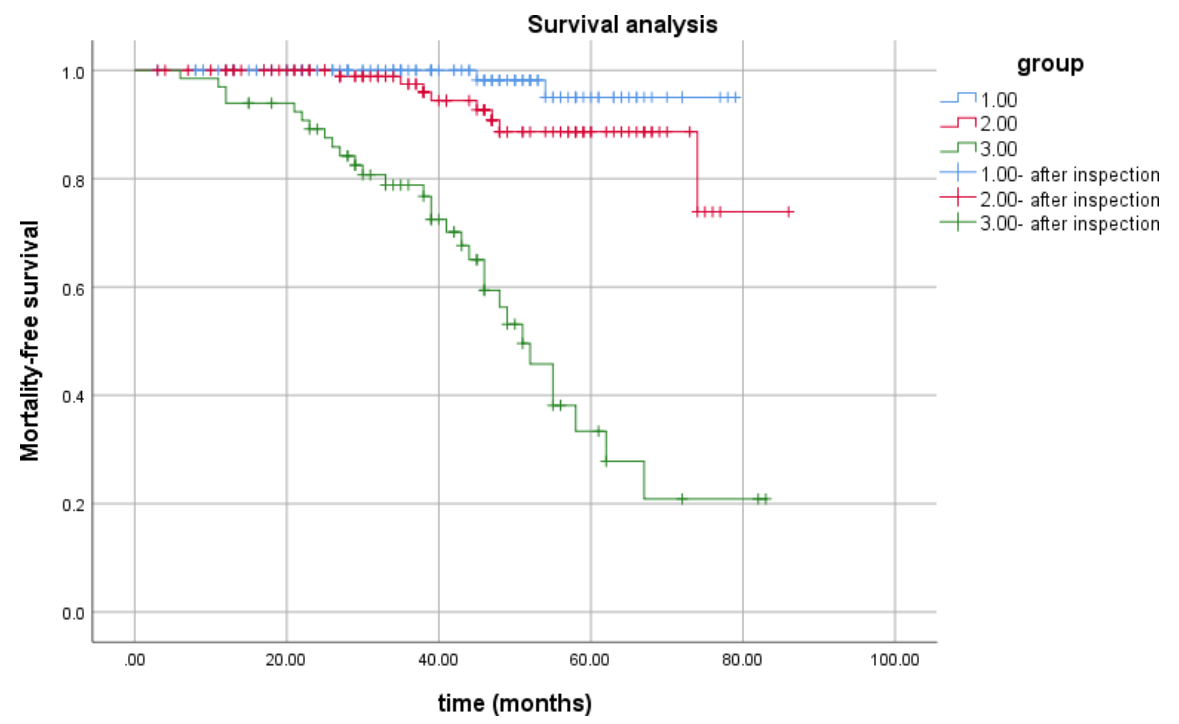

